\title{
VISCOSIDADES DE MISTURAS FORMADAS POR TRIACILGLICERÓIS, ÁCIDOS GRAXOS E ALCOÓIS.
}

\author{
P. M. FLORIDO ${ }^{1}$, D. P. S. LOBO ${ }^{1}$, G. A. FARIA ${ }^{1}$, K. K. ARACAVA ${ }^{1}$, C. E. C. RODRIGUES ${ }^{1}$, R. \\ CERIANI $^{2}$ e C. B. GONÇALVES ${ }^{1}$ \\ ${ }^{1}$ Universidade de São Paulo, Departamento de Engenharia de Alimentos \\ ${ }^{2}$ Universidade Estadual de Campinas, Departamento de Engenharia Química \\ E-mail para contato: cintiabg@usp.br
}

\begin{abstract}
RESUMO - Dados de viscosidade permitem aprimorar processos de obtenção e purificação de óleos vegetais utilizando solventes. Devido ao grande número de variáveis que afeta o processo, a aquisição de dados em todas as condições de interesse não é viável e métodos para estimá-las são fundamentais. O objetivo deste trabalho foi testar diferentes modelos preditivos para a viscosidade e compará-los com dados experimentais referentes a sistemas formados por triacilgliceróis (tricaprilina e tricaprina), ácidos graxos (láurico, palmítico e linoleico) e alcoóis de até 6 carbonos (etanol, isopropanol e hexanol). As viscosidades foram obtidas em um viscosímetro automático (Anton Paar). Para a predição dos dados experimentais, foram testadas as formas simples e modificada da Regra de Kay, além do modelo de contribuição de grupos GC-UNIMOD. Os resultados mostraram que, apesar da complexidade das misturas, os modelos mais simples são capazes de fazer uma boa predição da viscosidade, com desvios médios relativos (DMR) inferiores a $7 \%$, principalmente para misturas com maiores concentrações de solvente, podendo ser uma importante ferramenta para a indústria de óleos e gorduras.
\end{abstract}

\section{INTRODUÇÃO}

Óleos e gorduras, tanto de origem animal como vegetal, são insumos importantes nas áreas química, farmacêutica, cosmética, e alimentícia. São compostos principalmente por triacilgliceróis (TAG), podendo conter em menor proporção ácidos graxos livres, gliceróis parciais e outros componentes lipossolúveis como vitaminas. O estudo de sistemas-modelo contendo compostos graxos e solventes é de grande interesse industrial, pois possibilita, por exemplo, o aprimoramento nos processos de obtenção e purificação de óleos vegetais. A literatura reporta dados de viscosidade para compostos graxos puros, misturas-modelo dos mesmos, e óleos vegetais (Esteban et al., 2012; Pratas et al., 2011; Ceriani et al., 2007, Ceriani et al., 2008; Gonçalves et al., 2007; Fasina et al., 2006; entre outros), porém, sistemas contendo esses mesmos compostos e solventes alcoólicos foram pouco explorados (Edujee e Boyes, 1980; Rodríguez et al., 1994, Resa et al., 2002; Sanaiotti et al., 2010).

Há, portanto, uma escassez de dados de densidade e viscosidade para essas misturas-modelo, sendo necessário preencher essa lacuna devido à importância dessas propriedades para o projeto de equipamentos e tubulações envolvidos nos processos industriais. É preciso observar que nem sempre é viável medir as viscosidades e densidades em todas as condições de interesse, pois há uma gama de 
variáveis e suas combinações que afetam as propriedades das misturas. Consequentemente, métodos para estimar estas propriedades em diferentes condições são de grande importância prática (Ceriani et al., 2011). A literatura reporta alguns modelos para o cálculo da viscosidade, dentre eles, destacam-se os modelos baseados na fração molar, como a Regra de Kay (Azian et al., 2001), tanto na forma simples como modificada, e o CG-UNIMOD (Cao et al., 1993), que é baseado no conceito de contribuição de grupos.

Desta forma, o objetivo deste trabalho foi determinar dados experimentais de viscosidade de sistemas-modelo contendo triacilgliceróis, ácidos graxos e solventes alcoólicos, além de testar os modelos simples e complexos supramencionados para a estimativa desta propriedade física.

\section{MATERIAL E MÉTODOS}

\subsection{Preparo das misturas}

Para formar as os sistemas graxos, foram utilizados dois tipos de triacilgliceróis (tricaprilina e tricaprina, ambos da Nu-chek, pureza > $99 \%$ ), três tipos de ácidos graxos (láurico, palmítico e linoleico, todos da $\mathrm{Nu}$-chek, pureza > $99 \%$ ) e três tipos de solventes alcoólicos (etanol anidro, isopropanol e hexanol, todas da Merck, pureza > $99 \%$ ).

As viscosidades foram determinadas para os seguintes sistemas: tricaprilina + ácido linoleico + etanol (Sistema 1), de 20 a $60^{\circ} \mathrm{C}$ (com intervalos de $10^{\circ} \mathrm{C}$ ) e em três diferentes concentrações; tricaprina + ácido láurico + isopropanol (Sistema 2), de 30 a $60{ }^{\circ} \mathrm{C} \mathrm{(com} \mathrm{intervalos} \mathrm{de} 5^{\circ} \mathrm{C}$ ) e em duas diferentes concentrações; tricaprilina + ácido palmítico + hexanol (Sistema 3), de 30 a $70{ }^{\circ} \mathrm{C}$ (com intervalos de $10^{\circ} \mathrm{C}$ ) e em quatro diferentes concentrações; e tricaprina + ácido láurico + hexanol (Sistema 4), de 30 a $70^{\circ} \mathrm{C}$ (com intervalos de $10^{\circ} \mathrm{C}$ ) e em cinco diferentes concentrações. Como ao fato do etanol não ser completamente miscível em compostos graxos, as composições do sistema 1 foram escolhidas levando-se em consideração os dados de equilíbrio líquido-líquido disponíveis em Krip (2002). Para prevenir a separação de fases durante a medida experimental, as composições foram escolhidas próximas à curva binodal, porém na região monofásica. Para os outros sistemas, esse procedimento não foi necessário.

Cada componente foi pesado dentro de um tubo de polipropileno (15 mL, Corning Inc.), utilizando-se uma balança analítica (Adam, modelo PW 254), com precisão de 0,0001 g. Após a pesagem, os tubos foram devidamente fechados, agitados manualmente por aproximadamente 5 minutos, e deixados em repouso em banho termostático (Tecnal, modelo TE-2005) para atingir a temperatura de medição. As amostras foram retiradas dos tubos com o auxílio de seringas descartáveis e levadas para análise. 


\subsection{Determinação experimental}

As viscosidades cinemáticas foram determinadas em um viscosímetro automático (Anton-Paar, modelo AMVn), com controle de temperatura. O princípio da determinação é o tempo de efluxo de uma esfera imersa na amostra de um capilar de vidro devidamente calibrado. Como recomendado pelo fabricante, as determinações experimentais foram realizadas com um ângulo de inclinação de 70 ${ }^{\circ}$ em quadruplicate. Os desvios padrões das medidas variaram entre 0,0001 e 0,5749 mPa.s.

Como o equipamento de determinação de viscosidade requer a densidade da amostra como dado de entrada, esta propriedade também foi medida em um densímetro digital de bancada (Anton-Paar, modelo DMA4500). As amostras foram inseridas no equipamento com o auxílio de seringas descartáveis, sendo que cada análise foi realizada em triplicada, obtendo-se desvios padrões inferiores a $0,01 \mathrm{~kg} \cdot \mathrm{m}^{3}$.

\subsection{Estimativa das viscosidades}

Dois tipos de modelos foram utilizados para a estimativa das viscosidades. O primeiro deles considera apenas a composição da mistura e a viscosidade dos componentes puros, e inclui uma aproximação linear (Regra de Kay Simples) e sua forma logarítmica (Regra de Kay Modificada, Azian et al., 2001), apresentadas nas Equações 1 e 2, respectivamente.

$$
\begin{aligned}
& v_{\text {mistura }}=\sum_{i=1}^{n} c_{i} \cdot\left(v_{i}\right) \\
& \ln (v)_{\text {mistura }}=\sum_{i=1}^{n} c_{i} \cdot \ln \left(v_{i}\right)
\end{aligned}
$$

Nas Equações 1 e 2, o termo $v$ representa a viscosidade cinemática e $c_{i}$ representa a concentração do componente $i$ na mistura contendo $n$ compostos, que foi expressa em fração molar, $x_{i}$, ou fração mássica, $w_{i}$.

Como já mencionado, os modelos supramencionados (Equações 1 e 2) requerem o conhecimento da viscosidade de cada composto formador da mistura. Para os solventes alcoólicos, esses dados foram determinados experimentalmente. No entanto, devido ao elevado custo dos compostos graxos puros (triacilgliceróis e ácidos graxos), optou-se por utilizar o método de estimativa da viscosidade desenvolvido por Ceriani et al. (2011), o qual proporcionou desvios globais menores do que $5 \%$ para esses reagentes. Como modelo de Ceriani et al. (2011) fornece o dado em viscosidade dinâmica, utilizou-se o modelo de Halvorsen et al. (1993) para a predição da densidade $(\rho)$ dos compostos puros, possibilitando a conversão para viscosidade cinemática através da Equação 3.

$$
v=\frac{\eta}{\rho}
$$




\section{9 a 22 de outubro de 2014 \\ Florianópolis/SC}

O segundo tipo de modelo estudado, GC-UNIMOD (Cao et al., 1993), é baseado no conceito de contribuição de grupos, capaz de estimar as propriedades de uma mistura, tratando-a como uma solução de grupos funcionais das moléculas que as constituem. Sendo assim, cada propriedade física ou química pode ser considerada como a soma das contribuições feitas por cada um destes grupos e por suas interações. Esta propriedade aditiva, característica dos métodos de contribuição de grupos, é a grande vantagem dos modelos baseados neste conceito, pois a quantidade de informações necessárias para determinar as propriedades de uma mistura qualquer pode ser bem menor do que se fossem consideradas as contribuições de cada um de seus possíveis componentes. De fato, o número de grupos funcionais é bem menor comparado ao número de moléculas de interesse neste trabalho.

Para a utilização do GC-UNIMOD, é necessário o conhecimento dos parâmetros de interação entre os grupos funcionais presentes nas moléculas da mistura de interesse. A literatura já apresenta um vasto número de parâmetros de interação entre vários pares de grupos funcionais que compõem as misturas estudadas: $\mathrm{CH}_{3}, \mathrm{CH}_{2}, \mathrm{CH}, \mathrm{CH}=\mathrm{CH}, \mathrm{CH}_{2} \mathrm{COO}, \mathrm{OH}$ e $\mathrm{COOH}$.

O GC-UNIMOD (Group-Contribution Thermodynamics-Viscosity Model) é semelhante ao método UNIFAC proposto para predição do equilíbrio de fases, que leva em consideração duas contribuições para a viscosidade cinemática da mistura ( $\left.v_{\text {mistura }}\right)$ : uma parte combinatorial e outra residual, como mostra a Equação 4.

$\ln \left(v_{\text {mistura }}\right)=\sum_{i=1}^{n}\left[\xi_{i}^{C}+\xi_{i}^{R}\right]$

A parte combinatorial, $\xi_{i}^{C}$, considera as diferenças no tamanho das moléculas presentes na mistura, de modo que somente as propriedades das substâncias puras são necessárias para o cálculo. O termo residual, $\xi_{i}^{R}$, é mais complexa e leva em consideração a energia de interação entre os pares de grupos funcionais presentes na mistura. A completa descrição deste modelo pode ser encontrada em Gonçalves et al., 2007). Os valores de $R_{k}$ (parâmetro de volume do grupo $k$ ) e $Q_{k}$ (parâmetro de área do grupo $k$ ), presentes nos termos combinatorial e residual, respectivamente, foram obtidos de Fredenslund e Sorensen (1994). Os parâmetros de interação entre os grupos funcionais foram obtidos de Larsen et al. (1987).

\section{RESULTADOS E DISCUSSÃO}

O desempenho dos modelos testados para o cálculo das viscosidades pode ser visualizado na Tabela 1 a seguir, que apresenta os valores dos desvios médios relativos $(D M R)$ para todos os sistemas, calculado de acordo com a Equação 5. Vale ressaltar que a estimativa das viscosidades através das Equações 1 e 2 foram conduzidas considerando-se o termo ci tanto em fração molar como em fração mássica.

$$
D M R=100\left(\sum_{i=1}^{n} \frac{\left|v_{\text {calc }}-v_{\text {exp }}\right|}{v_{\text {exp }}} / N\right)
$$


Tabela 1 - Desvios médios relativos (DRM, em \%) para as viscosidades.

\begin{tabular}{|c|c|c|c|c|c|c|}
\hline \multicolumn{2}{|c|}{ Sistema } & Modelo A* & Modelo B* & Modelo C* & Modelo D* & Modelo E* \\
\hline \multirow{3}{*}{1} & $w_{\text {etanol }}=0,4882$ & 6,04 & 175,78 & 43,27 & 48,61 & 76,66 \\
\cline { 2 - 7 } & $w_{\text {etanol }}=0,6542$ & 23,12 & 111,90 & 47,58 & 4,37 & 72,88 \\
\cline { 2 - 7 } & $w_{\text {etanol }}=0,9149$ & 4,56 & 62,42 & 13,43 & 3,60 & 26,19 \\
\hline \multirow{3}{*}{2} & $w_{\text {isopropanol }}=0,6783$ & 6,88 & 75,95 & 25,30 & 16,13 & 25,01 \\
\cline { 2 - 7 } & $w_{\text {isopropanol }}=0,8211$ & 11,16 & 44,38 & 21,83 & 1,26 & 20,68 \\
\hline \multirow{4}{*}{3} & $w_{\text {hexanol }}=0,6243$ & 11,96 & 63,19 & 3,89 & 30,99 & 17,71 \\
\cline { 2 - 7 } & $w_{\text {hexanol }}=0,7596$ & 12,98 & 53,64 & 2,75 & 26,52 & 18,57 \\
\cline { 2 - 7 } & $w_{\text {hexanol }}=0,7785$ & 6,48 & 42,71 & 2,82 & 18,37 & 11,40 \\
\cline { 2 - 7 } & $w_{\text {hexanol }}=0,9177$ & 6,26 & 22,20 & 2,71 & 10,93 & 8,48 \\
\hline \multirow{4}{*}{4} & $w_{\text {hexanol }}=0,3436$ & 11,52 & 31,13 & 25,01 & 13,97 & 13,27 \\
\cline { 2 - 7 } & $w_{\text {hexanol }}=0,6011$ & 2,84 & 50,19 & 8,51 & 25,17 & 6,41 \\
\cline { 2 - 7 } & $w_{\text {hexanol }}=0,8280$ & 3,63 & 31,19 & 1,13 & 14,84 & 7,87 \\
\cline { 2 - 7 } & $w_{\text {hexanol }}=0,8749$ & 3,94 & 24,08 & 1,08 & 12,21 & 7,38 \\
\cline { 2 - 7 } & $w_{\text {hexanol }}=0,8970$ & 4,03 & 21,72 & 1,58 & 10,88 & 7,00 \\
\hline
\end{tabular}

*Modelo A = Regra de Kay Simples em fração molar; Modelo B = Regra de Kay Simples em fração mássica; Modelo C = Regra de Kay Modificada em fração molar; Modelo D = Regra de Kay Modificada em fração mássica; Modelo E = GC-UNIMOD.

De acordo com a Tabela 1, embora o GC-UNIMOD (Modelo E) seja um modelo mais detalhado, este provou ser incapaz de descrever as viscosidades de misturas complexas, contendo alcoóis com menor número de carbonos (etanol e isopropanol), apresentado valor de $D M R$ bastante elevados nesses casos. A Regra de Kay ponderada em fração mássica (Modelo B) também se mostrou não ser uma boa opção, com $D M R$ elevados para todos os sistemas. No entanto, este mesmo modelo ponderado em fração molar (Modelo A) forneceu valores de DMR menores do que 7 \% em 9 dos 14 sistemas estudados, e apresentou o melhor desempenho em 4 situações, correspondentes às menores concentrações de solvente (e consequentemente, maiores concentrações de compostos graxos) dos sistemas $1\left(w_{\text {etanol }}=0,4882\right), 2\left(w_{\text {isopropanol }}=0,6783\right)$ e 4 situações $\left(w_{\text {hexanol }}=0,3436\right.$ e $w_{\text {hexanol }}=$ 0,6011). Já a Regra de Kay Modificada, ponderada em fração molar (Modelo C), não foi capaz de descrever as viscosidades das misturas contendo etanol e isopropanol, porém, proporcionou os menores valores de DMR para 7 das 9 misturas contendo hexanol. O mesmo modelo, ponderado em fração mássica (Modelo D), mostrou-se a melhor opção para as misturas contendo etanol e isopropanol, porém somente para os menores teores de compostos graxos (equivalente aos maiores valores de fração mássica de solvente).

Nota-se que, com exceção do sistema 4 com $w_{\text {hexanol igual a } 0,3436}(D M R=11,52 \%)$, foi possível obter valores aceitáveis em termos de predição de viscosidade (DRM menores do que $7 \%$ ), utilizando-se pelo menos um dos modelos testados. De fato, esta mistura é a única na qual a concentração de compostos graxos supera a de solvente, indicando que os modelos testados não são adequados para essas situações.

As Figuras 1 a 4 apresentam os dados experimentais de viscosidade cinemática obtidos neste trabalho, além dos valores calculados pelo modelo que apresentou o melhor desempenho em cada mistura em particular. 

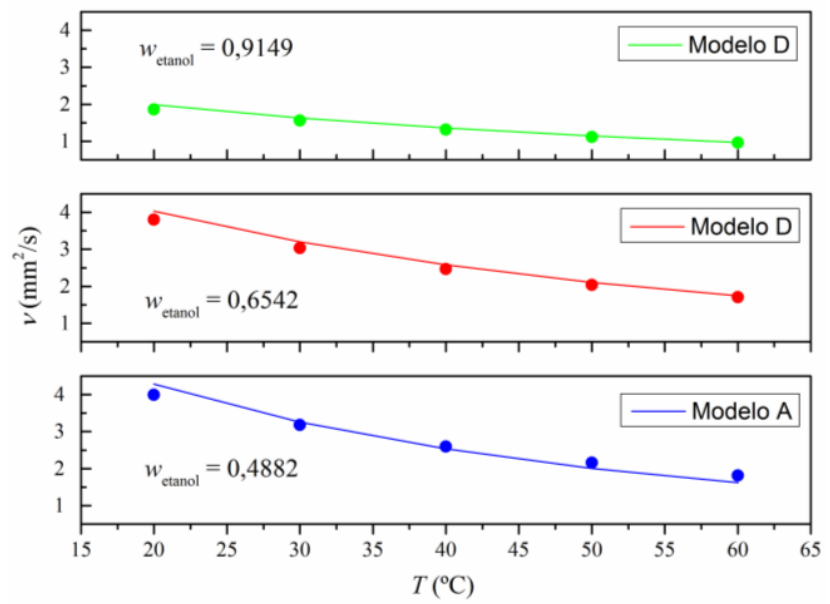

Figura 1 - Viscosidades cinemáticas ( $v$ ) para o sistema 1 (tricaprilina + ácido linoleico + etanol) em função da temperatura $(T)$ : Símbolos $=$ dados experimentais

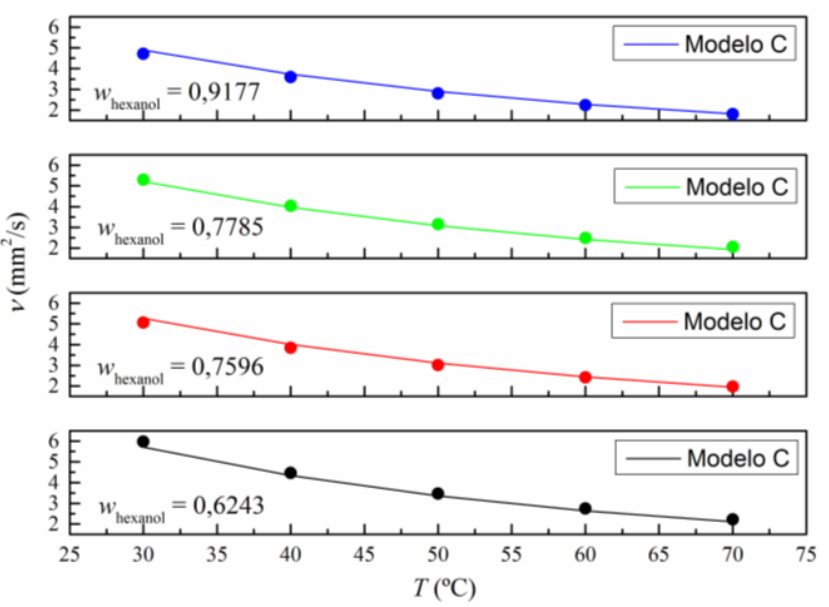

Figura 3 - Viscosidades cinemáticas ( v) para o sistema 3 (tricaprilina + ácido palmítico + hexanol) em função da temperatura $(T)$ :

Símbolos $=$ dados experimentais

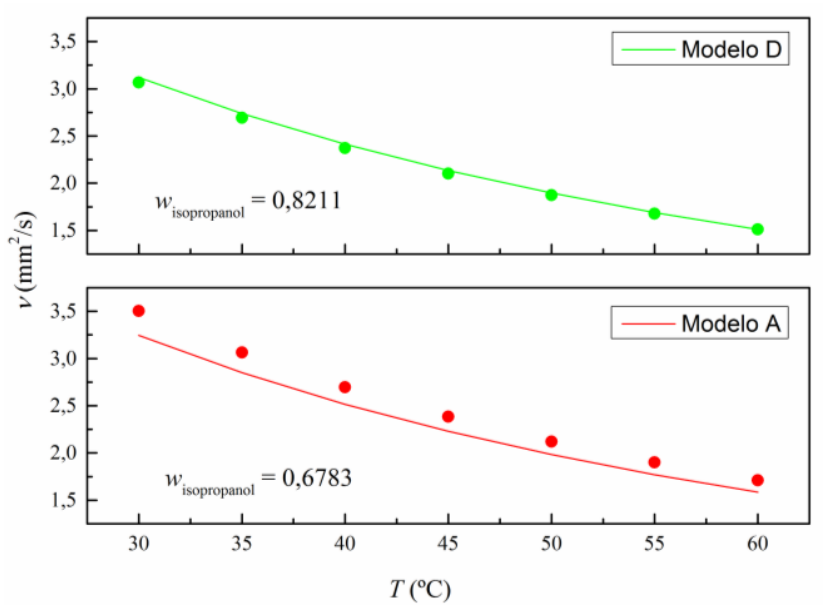

Figura 2 - Viscosidades cinemáticas ( $v$ ) para o sistema 2 (tricaprina + ácido láurico + isopropanol) em função da temperatura $(T)$ : Símbolos $=$ dados experimentais

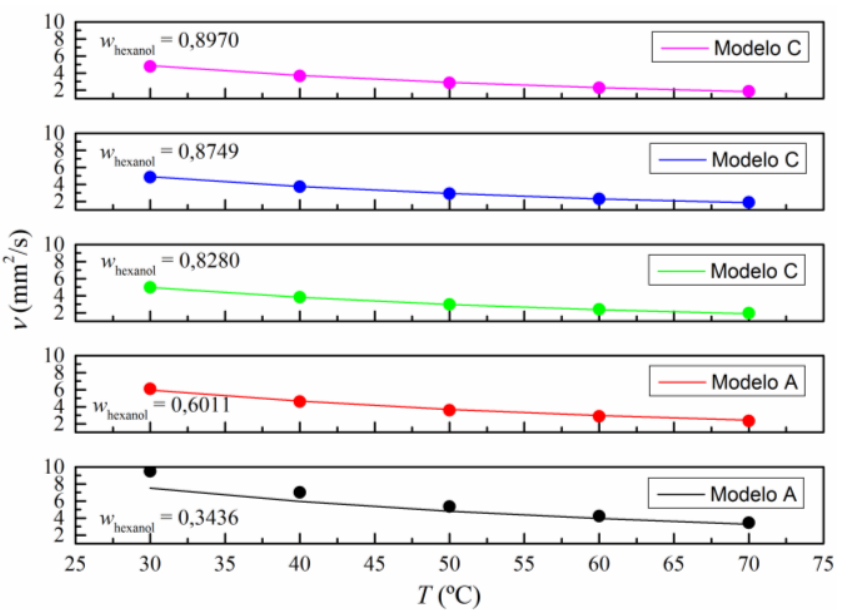

Figura 4 - Viscosidades cinemáticas ( $v$ ) para o sistema 4 (tricaprina + ácido láurico + hexanol) em função da temperatura $(T)$ : Símbolos $=$ dados experimentais 


\section{9 a 22 de outubro de 2014 \\ Florianópolis/SC}

Como esperado, as Figuras 1 a 4 mostram que a viscosidade tende a diminuir com o aumento da temperatura. De fato, a viscosidade está relacionada com as forces de atração entre as moléculas. Consequentemente, quando a energia cinética do sistema aumenta (decorrente do aumento da temperature), as moléculas permanecem menos tempo próximas umas das outras, tornando as forças intermoleculares menos efetivas, diminuindo a viscosidade.

\section{CONCLUSÃO}

O presente trabalho apresentou dados de viscosidade para misturas contendo triacilgliceróis, ácidos graxos e solventes alcoólicos, os quais foram utilizados para testar a capacidade preditiva de alguns modelos existentes na literatura. Os resultados mostraram que é possível estimar a viscosidade de misturas complexas, com concentração de compostos graxos (triacilgliceróis + ácidos graxos) inferiores à de solvente, utilizando modelos simples, baseados na composição do sistema e nas propriedades dos componentes puros. Porém, para misturas contendo grandes concentrações de compostos graxos, a obtenção de métodos mais eficaz ainda é um desafio. Desta forma, os dados apresentados neste trabalho podem ser um ponto de partida para a modelagem de parâmetros e para o desenvolvimento de novos modelos, tornando-se uma importante ferramenta no projeto de equipamentos para a indústria processadora de óleos e gorduras.

\section{AGRADECIMENTOS}

Os autores gostariam de agradecer à Fundação de Amparo à Pesquisa do Estado de São Paulo (FAPESP, Processo no 23203-1) pelo auxílio financeiro para a realização desta pesquisa.

\section{REFERÊNCIAS}

AZIAN, M. N.; KAMAL, A. A. M.; PANAU, F.; TEN, W. K. Viscosity Estimation of Triacylglycerols and of Some Vegetable Oils, based on their Triacylglycerol Composition. J. Am. Oil Chem. Soc., v. 78, p. 1001-1005, 2001.

CAO, W.; KNUDSE, K.; FREDENSLUND, A.; RASMUSSEN, P. Group-Contribution Viscosity Predictions of Liquid Mixtures Using UNIFAC-VLE Parameters. Ind. Eng. Chem. Res., v. 32, p. 2088-2092, 1993.

CERIANI, R.; GONÇALVES, C. B.; COUTINHO, J. A. P. Prediction of Viscosities of Fatty Compounds and Biodiesel by Group Contribution. Energy Fuels, v. 25, p. 3712-3717, 2011.

CERIANI, R; GONÇALVES, C. B.; RABELO, J.; CARUSO, M. W.; CUNHA, A. C. C.; CAVAlERI, F. W; BATISTA, E. A. C.; MEIRELLES, A. J. A. Group Contribution Model for Predicting Viscosity of Fatty Compounds. J. Chem. Eng. Data, v. 52, p. 965-972, 2007. 
CERIANI, R.; PAIVA, F. R.; GONÇALVES, C. B.; BATISTA, E. A. C.; MEIRELLES, A. J. A. Densities and Viscosities of Vegetable Oils of Nutritional Value. J. Chem. Eng. Data, v. 53, p. 1846-1853, 2008.

EDUJEE, G.H.; BOYES, A.P. Viscosity of some binary liquid mixtures of oleic acid and triolein with selected solvents. J. Chem. Eng. Data, v. 25, p. 249-252, 1980.

ESTEBAN, B.; RIBA, J. R.; BAQUERO, G.; RIUS, A.; PUIG, R. Temperature dependence of density and viscosity of vegetable oils. Biomass and bioenergy, v. 42, p. 164-171, 2012.

FASINA, O. O.; HALLMAN, H.; CRAIG-SCHMIDT, M.; CLEMENTS, C. Predicting Temperature-Dependence Viscosity of Vegetable Oils from Fatty Acid Composition. J. Am. Oil Chem. Soc., v. 83, p. 899-9032006.

FREDENSLUND, A.; SORENSEN, J. M. Group Contribution Estimation Methods, in Models for Thermodynamics and Phase Equilibria Calculations, edited by S.I. Sandler, Marcel Dekker, New York, 1994.

GONÇALVES, C. B; CERIANI, R.; RABELO, J.; MAFFIA, M. C.; MEIRELlES, A. J. A. Viscosity of Fatty Mixtures: Experimental data and prediction. J. Chem. Eng. Data, v. 52, p. 2000-2006, 2007.

HALVORSEN, J. D.; MAMMEL JR, W. C.; CLEMENTS, L.D. Density estimation for fatty acids and some vegetable oils based on their fatty acid composition. J. Amer. Oil Chem. Soc., v. 30, p. 875-880, 1993.

LARSEN, B. L.; RASMUSSEN, P.; FREDENSLUND, A. A modified UNIFAC groupcontribution model for prediction of phase equilibria and heats of mixing. Ind. Eng. Chem. Res., v. 26, p. 2274-2286, 1987

PRATAS, M. J.; FREITAS, S. V. D.; OLIVEIRA, M. B.; MONTEIRO, S. C.; LIMA, A. S.; COUTINHO, J. A. P. Biodiesel density: Experimental measurements and prediction models. Energy Fuels, v. 25, p. 2333-2340, 2011.

RESA, J. M.; GONZALEZ, C.; FANEGA, M. A.; ORTIZ DE LANDALUCE, S.; LANZ, J. Enthalpies of mixing, heat capacities, and viscosities of alcohol (C1-C4) + olive oil mixtures at 298.15 K. J. Food Eng., v. 51, p. 113-118, 2002.

RODRÍGUEZ, M.; GALÁN, M.; MUÑOZ, M. J.; MARTÍN, R. Viscosity of Triglycerides + Alcohols from 278 to 313 K. J. Chem. Eng. Data, v.39, p. 102-105, 1994.

SANAIOTTI G.; SILVA, C. A. S.; PARREIRA, A. G.; TÓTOLA, M. R.; MEIRELLES, A. J. A.; BATISTA, E. A. C. Densities, Viscosities, Interfacial Tensions, and Liquid-Liquid Equilibrium Data for Systems Composed of Soybean Oil + Commercial Linoleic Acid + Ethanol + Water at 298.2 K. J. Chem. Eng. Data, v. 55, p. 5237-5245, 2010. 